assume that it would be at least $100^{\circ}$ below the zero of Fahrenheit's scale, or $180^{\circ}$ below the actual mean temperature of the tropics. Of course, under such circumstances, the temperature of the tropics would be no higher than that of the Poles. Making this assumption, then, the oscillation of the temperature during the course of the sun-spot cycle is only I/I 80 of the total effect. Now the amplitude of the semi-diurnal element of the barometric oscillation at Batavia (the most equatorial of the three stations) is $1.896 \mathrm{~mm}$., or 0.0746 of an inch, and the $1 / 180$ part of this is $0^{\circ} 0004$ inch-a quantity that would be quite inappreciable in an investigation of this kind, masked as it must be by the much larger irregular variations shown by the register. It does not seem, then, that Dr. Hann's negative results really affect the validity of the positive evidence already afforded by other meteorological phenomena, and need not discourage us in our endeavours to obtain an explanation of the paradox indicated above, which, to my mind, is the most interesting feature of the problem. HENRY F. BLANFORD.

\section{THE QUESTION OF THE ASTEROIDS.}

$\mathrm{I}^{\mathrm{T}}$

has already been noted that the editors of the Berliner Fahrbuch have decided only to issue ephemerides for certain of the minor planets (NATURE, vol. xliii. p. III). The Bureau des Longitudes has endeavoured to remedy the inconvenience that arises from this decision by an extension of one of its departments. The general discussion that led the Bureau to adopt this course, and the importance of observations of these comparatively small bodies, are well expounded by M.F. Tisserand in the Annuaire for I89I.

Kepler recognized the continuity of the mean distances of the planets from the sun, when he said : "Infra Martem et Jovem novum interposui planetam." The publication of Bode's empirical law in I772 confirmed Kepler's ideas, and fixed the distance of the hypothetical planet as $2 \cdot 8$ times the mean distance of the earth from our luminary. But the existence of such a planet appeared still more probable when the calculations of Lexell and Laplace had shown that the magnitude of the orbit of the planet Uranus, discovered by Sir William Herschel in $\mathrm{I} 78 \mathrm{I}$, might have been predicted with accuracy from this relation between planetary distances. At a Congress held at Gotha in I796, it was proposed to search for the unknown body, and twenty-four astronomers each undertook the examination of an hour of the zodiac. The discovery of Ceres by Piazzi on January I, I80I, almost before the association of observers had got fairly to work, is a matter of common knowledge. Gauss's calculations showed that the mean distance of this planet from the sun is 277 , which corresponds with that indicated by Bode's law; hence the gap appeared to be filled, but by a body of very modest size, for the measures made by Herschel only assign it a diameter of about I 55 miles.

Olbers's discovery of a second planet, Pallas, moving round the sun at the same mean distance as Ceres, gave the question another aspect. It was proved by Gauss that the two bodies may pass very near to each other at two points situated on the line of intersection of the planes of their orbits. This led Olbers to oelieve that the new planets were portions of a larger body broken up by some internal disturbance, and he accordingly suggested that other fragments might be found near the points of intersection of their orbits. The hypothesis was supported by Harding's discovery of Juno in 1804 , near one extremity of the line of intersection, and the discovery of Vesta by himself in 1807 , close to the other extremity.

It was not until i 845 that a fifth planet was discovered by Encke, and this was even smaller than the four that preceded it. After this date the discoveries became more frequent, and now the number has reached 308 . But the magnitudes of the newly-discovered bodies are decreasing, for, whilst the first four have magnitudes comprised between 6 and 8, the two discovered by Encke are only of the 9 th magnitude, and those now found are rarely brighter than the $13^{\text {th }}$ magnitude.

The hypothesis advanced by Olbers as to the origin of the asteroids - a designation due to Herschel-has not found much support. Newcomb, from an investigation of the orbits of the first forty asteroids, found that their planes are far from having a common line of intersection. It may be suggested that this geometrical condition was fulfilled at a certain epoch, and that the perturbations caused by Jupiter and Saturn have caused the present distribution. Calculations show, however, that the required condition never existed; hence Olbers's hypothesis must be abandoned.

With regard to the width of the zone which contains the orbits of the asteroids, we find that (149) moves round the sun at the shortest mean distance, viz. $2 \cdot 13$, and that (279), the asteroid most removed from our luminary, has a mean distance of $4^{2} 26$. The periods of revolution of these two bodies are, respectively, 3.11 and 8.81 years. It will therefore be seen that the asteroids revolve in orbits much greater and less than that assigned by Bode's law.

When the eccentricities of the orbits are considered, it is found that (132) may approach to a distance of $I .6 I$ from the sun, whilst $(17$ may get so far away as 4.73 times the earth's mean distance. The asteroids are, therefore, contained in a wide zone, and the whole of their positions form a kind of ring having a radius a little more than three times the distance of the earth from the sun.

If the asteroids are arranged into groups, of which the eccentricities are comprised between limits differing by 0.05 , the following result is obtained :-

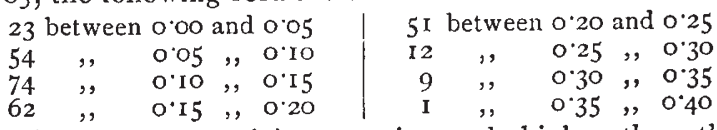

The mean eccentricity, 015 , is much higher than the corresponding mean of the major planets-viz. 0.86 . It appears, therefore, that some notable differences must have existed in the conditions of formation.

But the difference is still more striking in the case of the inclinations of the orbits. The mean inclination is $8^{\circ}$, which is slightly greater than that of Mercury and that of the sun's equator. Of 293 asteroids, 17 have inclinations greater than $20^{\circ}$. These are given in the following table, and also their eccentricities and mean distances:-

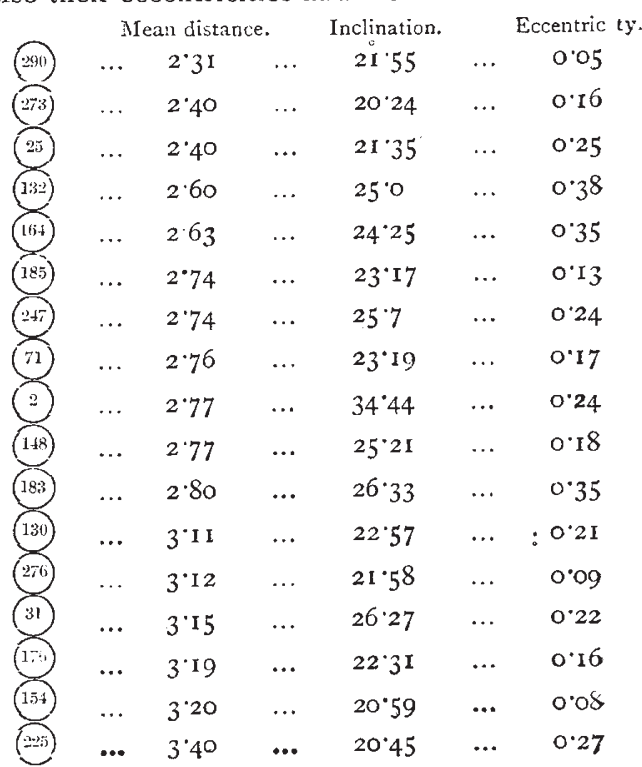

NO. I 12 I, VOL. 43 ] 
It will be seen that, with two exceptions, the orbits greatly inclined to the ecliptic have also considerable eccentricity. The converse of this is not true, however, for great eccentricity does not appear necessarily to carry with it a high inclination. A consideration of these eccentricities and inclinations naturally leads one to ask whether they were the same at the time of the formation of the bodies whose orbits they represent, or whether the values have been increased under the influence of perturbations. On this point Leverrier made the following remark:-

"There exists a region between Jupiter and the sun, such that, if a small mass could be placed there, in an orbit slightly inclined to that of Jupiter, this little mass would be able to move out of its primitive orbit, and to attain a great inclination to the plane of the orbit of this planet and to that of Saturn. It is remarkable that this position is found at very nearly double the distance of the earth from the sun-that is to say, at the interior limit of the zone where the minor planets are found."

This fact is very interesting in itself, but it is not sufficent to explain the large inclinations that are found at the distances 2.75 and 3.15 . M. Tisserand found some years ago that the region of instability is at a distance of only 1.83 from the sun. We must therefore conclude that the perturbations caused by Jupiter and Saturn are insufficient to explain the considerable values of the eccentricities and inclinations of a great number of asteroids; these values are never very small, and consequently the conditions under which Laplace's hypothetical nebulosity existed were not the same at the time of formation of the older planets as at the creation of the asteroids. The question is therefore a very interesting one from a cosmographical point of view, and the accumulation of new discoveries of asteroids is the only thing that will facilitate its solution.

The distribution of asteroids according to their mean distances from the sun, or, what amounts to the same thing, according to their mean diurnal motion, brings out some interesting facts. If the number of asteroids be tabulated having mean movements comprised between $540^{\prime \prime}$ and $55^{\prime \prime}, 50^{\prime \prime}$ and $560^{\prime \prime}$, and so on, with increments

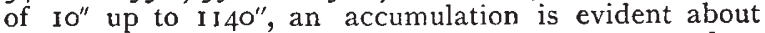
$640^{\prime \prime}, 780^{\prime \prime}$, and 8I $5^{\prime \prime}$, which movements correspond to the mean distances $3.13,275$, and 267 . Two gaps are seen about $60^{\prime \prime}$ and $900^{\prime \prime}$, or at the distances 3.27 and 2.50 . The mean diurnal motion of Jupiter is $299^{\prime \prime} \cdot 12$, or nearly $300^{\prime \prime}$. It will therefore be seen that the gaps correspond to two regions where the mean motion of the planet would be exactly double or triple that of Jupiter. There are other gaps less definitely marked, where the relation between the two diurnal motions, instead of being equal to 2 or 3 , is represented by the fractions $\frac{5}{3}, \frac{7}{3}$, $5, \frac{7}{2}$, \&c. Kirkwood developed this relation in 1866 , and generalized it by saying that the parts of the zone of the asteroids in which there exists a simple commensurable relation between the time of revolution of a minor planet and that of Jupiter are represented by gaps similar to the intervals that separate the different rings of Saturn. It should be remarked, however, that the intervals are not so well defined as in the case of Saturn's ring, inasmuch as after a gap the number of asteroids does not increase sharply, but little by little, until it reaches the normal value.

Can these gaps be explained by the theory of perturbations? The illustrious Gauss, writing to Bessel in $18 \mathbf{1} 2$, remarked: "The mean motions of Jupiter and Pallas are in the relation expressed by $7^{7} 8$, a value that ought to be realized more and more exactly under the influence of the attraction of Jupiter, as is also the case in the equality of the motions of revolution and rotation of the moon."

Newcomb in his researches into Saturn's system, found that, in the case of exactly commensurable motions, the perturbations could not increase beyond a. certain limit.
But this consequence is by no means necessary, for, in all probability, there would only be more or less irregular oscillations, and equilibrium would then be restored. The work of Gylden and $M$. Tisserand himself tend to the same conclusion. It is therefore probable that if the gaps had not existed in the beginning, the ulterior perturbations by Jupiter would not have been sufficient to produce them ; they must have existed immediately after the formation of the asteroids. This is another reason why the question is of interest from a cosmographical point of view.

The asteroids situated at the outer limit of the ring are not devoid of interest. Some of them have orbits very similar to certain comets of short period; thus the orbit of 175 is very similar to that of Tempel's periodic comet (1867 II.), as is shown by the following comparison :--

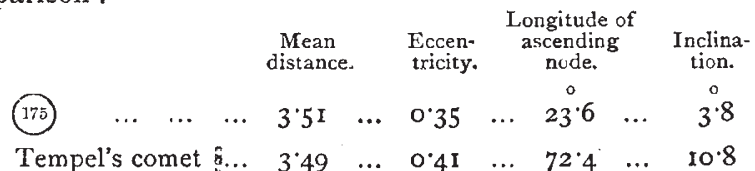

Other asteroids are important because of their near approach to Jupiter. Of these, 279, discovered by Palisa about two years ago, is of special interest, for in 1912 it will be at the same distance from Jupiter as the earth is from the sun. At this time the attraction of Jupiter on the asteroid will be more than $\frac{1}{5}$ that of the sun; thus the calculations of the perturbations promise to be interesting and difficult, and from them the mass of Jupiter may be determined with considerable precision.

The asteroids which occur at the inner limit of the ring are also useful, especially if their orbits are very eccentric and they approach the earth within 0.7 its mean distance. In such cases their parallax may be very accurately determined by observations made at two stations some distance apart. From the values obtained, the parallax of the sun may be deduced, and this is one of the best methods at our disposal for the determination of an element of fundamental importance in astronomy.

We have said that it is impossible to entertain the idea that all the asteroids were formed by the rupture of a single planet. But groups of two planets may be formed having strikingly analogous elements. The most interesting is formed by the asteroids (37) and (66); their orbits are almost equal ellipses, situated very nearly in the same plane, and differ only in the orientation of their major axes. The analogy is apparent from the following comparison of elements :-

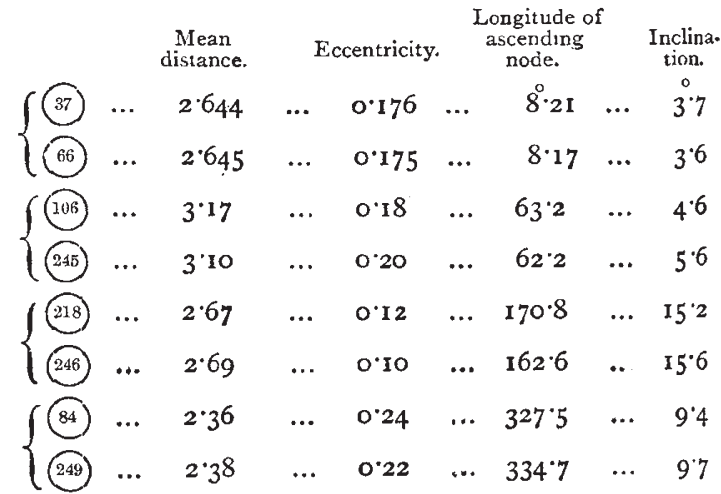

This quasi-identity of elements cannot be accidental, and it would not require many facts of this nature to throw new light upon the origin and formation of the bodies possessing them.

Enough has been said to prove that the discovery of NO. I I 2 I, VOL. 43 ] 
asteroids has brought out some important facts. For this and other reasons, $M$. Tisserand thinks that the search for them should be continued. The calculations required to furnish elements and ephemerides are certainly formidable, but they could be divided among several scientific establishments. The Burreau des Longitudes is willing to play an onerous part. We hope that sufficient resources will be accorded to it to allow this extremely useful work to be carried on. RICHARD A. GREGORY.

\section{SOLUTIONS.}

THE brilliant presidential address of Prof. Orme Masson at the Chemical Section of the Australasian Association for the Advancement of Science marks a distinct advance in our ideas of solution. The analogy between the behaviour of a liquid and its vapour in presence of each other and of a pair of solvents capable of mutual solution is so striking as to carry conviction. The resemblance of the liquid-vapour curve, with its apex at the critical point, to the solubility curve, with its apex at the critical solution point, appears to me to prove beyond cavil that the two phenomena are essentially of the same nature.

There are two other phenomena, which, it appears to me, are made clear by the ideas of Prof. Masson. The first of these has reference to supersaturated solutions. The curves (published in NATURE, February 12, p. 348) showing the analogy between liquid-gas and solution curves, are isobaric curves, or, more correctly, they represent the terminations of isobaric curves in the region of mixtures, where, on the one hand, a liquid exists in presence of its vapour, and, on the other, one solvent in presence of another (for both solvents play the part of dissolved substances, as well as of solvents). $M$. Alexéeff's data are not sufficient to permit of the construction of a curve representing a similar region mapped out by the termination of isothermal lines. But it is obvious that it would be possible to determine osmotic pressures of various mixtures by the freezingpoint method, and so to construct isothermal curves for such mixtures of solvents. And there can be no reasonable doubt that, as the isobaric curves of liquid-gas and of solvent-solvent display so close an analogy, the isothermal curves would also closely resemble each other.

Granting, then, that this is the case, we may construct an imaginary isothermal curve on the model of the curve for alcohol published in the Phil. Trans. by Dr. Sydney Young and myself. Now, in one series of papers on the liquid-gas relations, we showed that with constant volume pressure is a linear fun ction of temperature ; and we were thus able to calculate approximately the pressures and volumes for any isothermal representing the continuous transition from the gaseous to the liquid state (see Phil. $M a g$., I 887 , vol. xxiii. p. 435). It would be interesting to ascertain whether, if concentration be kept constant, osmotic pressure would also show itself to be a linear function of temperature. But, this apart, it appears in the highest degree probable that there should also exist, in theory at least, a continuous transition from solvent to solvent, the representation of which would be a continuous curve. In such a case, on increasing the concentration of the solution by eliminating one solvent, the other solvent should not separate visibly, but the two should remain mixed until one solvent has been entirely removed. The accompanying diagram will make this clear. The sinuous curve $\mathrm{ABCDE}$ may represent either continuous change from gas to liquid along an isothermal on decrease of volume, or it may represent a similar continuous change from saturated solution to dissolved substance on increase of concentration.

I "Some Suggestions regarding Solutions." By Williain Ramsay, Ph. D., F.R.S., Professor of Chemistry in University College, London. Read before the Royal Society on Thursday, March 5 .
Mr. Aitken's experiments on the cooling of air con taining water-vapour have shown us that it is possible to realize a portion of the curve $A B$; the phenomenon of "boiling with bumping" constitutes a practical realization of a portion of the curve $\mathrm{DE}$; and we may profitably inquire what conditions deternine such unstable states with solvent and solvent.

Regarding the portion of the curve $A B$, I think that no reasonable doubt can be entertained. It precisely corresponds to the condition of supersaturation. In the liquid-gas curve, the volume is decreased at constant temperature without separation of Jiquid; in the solventsolvent curve the concentration is increased without separation of the solvents. Dr. Nicol has shown that it is possible to dissolve dry sodium sulphate in a saturated solution of sodium sulphate to a very considerable extent without inducing crystallization; and here we have a realization of the unstable portion of the curve $A B$. In the gas-liquid curve pressure falls with formation of a shower of drops ; in the solvent-solvent curve crystallization ensues, and the solvents separate. The phenomena are, however, not completely analogous; the complete analogy would be if the temperature were so low that the substance in the liquid-gas couple were to separate in the

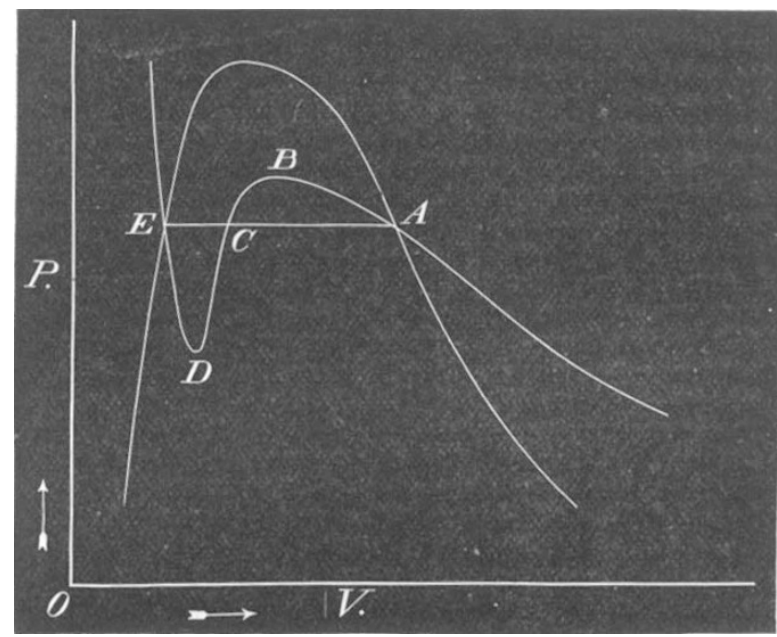

solid, not in the liquid, state. This, so far as I am aware, has not been experimentally realized, but one sees no reason why it should not be possible.

I have some hesitation in offering speculations as to the state of matter at the portion of the continuous curve $\mathrm{DE}$. It may be that it corresponds to a syrupy or viscous state. Cane-sugar at a moderate temperature dissolves water ; indeed it is possible to obtain a solution of I per cent. of water in molten cane-sugar. And such a solution, if quickly cooled, remains a syrup. But it can be induced to crystallize by the presence of crystals. Thus, in such a mixture of sugar and water, a few grains of crystalline sugar cause the whole mass to crystallize, and water saturated with sugar and sugar separate into two layers. Here, again, a complete analogy fails us, for it is a solid which separates. As we know nothing of the osmotic pressure of a syrup, the analogy is a defective one; but it is probable that a dilute solution of sugar would pass continuously into a syrup of pure sugar by evaporation of the solvent, and analogy would lead to the supposition that the syrup coincides with the unstable state of the liquid. I would, therefore, offer the analogy between the syrupy and the supercooled states as a tentative one : it lacks foundation in both cases.

One point remains to be mentioned. I have for the

NO. I I 2 I, VOL. 43] 